\title{
Impact of Road Bends on Traffic Flow in a Single-Lane Traffic System
}

\author{
Zeng Junwei, ${ }^{1}$ Qian Yongsheng, ${ }^{1}$ Xu Dejie, ${ }^{1}$ Jia Zhilong, ${ }^{1}$ and Huang Zhidan ${ }^{2}$ \\ ${ }^{1}$ School of Traffic and Transportation, Lanzhou Jiaotong University, Lanzhou 730070, China \\ ${ }^{2}$ School of Mechatronic Engineering, Lanzhou Jiaotong University, Lanzhou 730070, China \\ Correspondence should be addressed to Qian Yongsheng; qianyongsheng@mail.lzjtu.cn
}

Received 26 September 2013; Revised 5 December 2013; Accepted 19 December 2013; Published 4 February 2014

Academic Editor: Wuhong Wang

Copyright (c) 2014 Zeng Junwei et al. This is an open access article distributed under the Creative Commons Attribution License, which permits unrestricted use, distribution, and reproduction in any medium, provided the original work is properly cited.

\begin{abstract}
Taking the characteristics of road bends as a research object, this work proposes the cellular model (CA) with road bends based on the NaSch model, with which the traffic flow is examined under different conditions, such as bend radius, bend arc length, and road friction coefficiency. The simulation results show that, with the increase of the bend radius, the peak flow will be continuously increased, and the fundamental diagram will become more similar to that of the classic NaSch model; the smaller the bend radius is, the easier it is for the occurrence of blockage; for different bend lengths, all the corresponding traffic flows show that the phenomenon of go-and-stop and the bends exert slight inhibitory effect on traffic flow; under the same bend radius, the inhibition effect of the bends on the traffic flow will be weakened with the increase of the friction coefficiency.
\end{abstract}

\section{Introduction}

The contradiction between the road line and the limitation of the actual terrain (topography, towns and villages, natural lakes and rivers, etc.) should be fully considered in expressway. In addition, these factors should be taken into account comprehensively to ensure driving comfort, alignment continuity, easy operating, and road safety. Therefore, such elements as straight line, transition curve, circular curve, superelevation, and longitudinal grade should be taken into full consideration in the road line design so as to meet the design requirements to the greatest scale. It appears to be particularly an urgent issue to research the traffic flow characteristics on bends, to establish models for the simulation of vehicles on bends, and to analyze the impact of bends on road traffic flow.

The theory of cellular automata has been widely used in the field of road traffic flow. The CA models for traffic flow mainly include NaSch model, FI model, and BML model. It is originally based on the Wolfram cellular automata models [1]. NaSch model is a one-dimensional CA model, which can successfully simulate the go-and-stop traffic wave as well as the traffic flow transformation from free phase to congestion one. As to different factors, a variety of valuable traffic flow CA models have been established based on NaSch model [2]. Ishibashi and Fukui investigated dynamic effect of deceleration in advance on traffic flow [3]. Biham et al. researched the influence of headway space on traffic flow [4]. Qian et al. proposed the cellular automaton model that considers lane-control and speed-control to research the characteristics of highway traffic flow [5]. Yang et al. use the cellular automaton model to study the electric vehicle's electricity energy consumption under the different conditions [6]. Tang et al. proposes a cellular automaton (CA) model for dynamic simulations under mixed traffic conditions [7-10]. Ge et al. put some new CA model for intelligent transportation system, which makes the experiences for CA model of trains system [11, 12]. Li et al. propose a cellular automata model to simulate the traffic flow to analyze how the length of speedlimited section, train running time interval, and the speedlimit value affect the traffic flow. The decrease of the length of speed-limited section, the moderate increase of train running time interval, and the increase of the speed-limit value can improve the green light running time of the trains [13-16].

Currently, research work of traffic flow on road bends are limited. They are mainly conducted from the viewpoint 
of bend alignment combined with road line design. In 1995, Rao studied the alignment design of road bends [17], where the limit value of the bend radius, the tangent length of the transition curve, and the curvature change were discussed emphatically. Bentley and Gallagher explored the setup of the transition curve and made a summarized analysis on the bend alignment design of the US based on horizontal and vertical curve [18]. Dabbour et al. examined the vertical curve of the bends nonlinearly, proposed a road safety evaluation method, and analyzed the values of vertical curves for different alignment types [19]. Persaud et al. presented the guidance law for the hazard detection of road bends, pointing out that it is a low-cost traffic safety improvement measure with obvious effect to set effective bend hazard warnings [20].

Research works of road bends in China today are mainly conducted from the viewpoint of alignment design. $\mathrm{Mu}$ and Yang introduced the concept of possible velocity into road alignment design, providing a theoretical basis for the selection of highway design technical indicators by using possible velocity prediction method [21]. Liang and Xue examined the effect of curvature, arc length, and the road surface friction coefficiency on traffic flow and reproduced the phenomenon of go-and-stop in actual traffic conditions [22]. Wang et al. revealed the characteristics and laws of running speed on different types of roads and its relationship with horizontal curve radius through the analysis of different types of road running speed prediction models and pointed out horizontal curve design values for all levels of roads [23].

In summary, many domestic and foreign scholars have largely improved the study of road traffic flow or make a good progress at the aspect of CA model, but there are few studies on bend road by CA model. This work conducts further studies on the characteristics of traffic flow on road bends on the basis of the previous studies and makes supplement and perfection to the relevant theories, such as improved algorithm of NaSch model which is used on road bends. It will make a good development for the CA using on the traffic flow.

\section{Characteristics of Road Bends}

2.1. Characteristics of Road Bends Alignment Elements. The horizontal alignment includes straight line, circular curve, and transition curve. When traveling on straight line section, the vehicle is easy to control with better comfort. Thus the driver will be able to travel at high desired speed with little fluctuation. The transition curve located between straight line and circular curve plays a transition role; when traveling on transition curve section, the vehicle will accelerate when entering straight road section, will decelerate when entering circular curve section, and will enter the next section with appropriate speed. The length and radius of the circular curve have direct impacts on vehicle speeds: the smaller the radius, the worse the stability and the harder it is to manipulate the vehicle. As a result, the running speed will be reduced generally. On a circular curve section, the vehicle will be traveling at a constant speed and will be accelerated or decelerated at the end of the horizontal curve. When the radius of the circular curve is greater than the critical value, the impact of curvature on vehicle speed will disappear, and the circular curve can be treated as a straight line theoretically.

The vertical alignment mainly includes longitudinal gradient and vertical curve. The length and grade of the longitudinal gradient have a great impact on vehicle speed: under normal circumstances, the greater the length and grade, the lower the vehicle speed. Vertical curve is a circular curve connecting two longitudinal gradients, the purpose of which is to ensure smooth transition from one longitudinal gradient to the other; compared to the longitudinal gradient, a vertical curve has less impact on the vehicle speed.

A curved ramp is the connection of horizontal curve and longitudinal gradient, in which the radius of the horizontal curve $r \in[125,1000]$ and the grade of the longitudinal gradient $i \in[-6 \%, 2 \%]$. The curved ramp is a road accident blackspot, and its design quality must be critical enough to ensure traffic safety.

2.2. Characteristics of Traffic Flow on Road Bends. Road bends are the special sections of an expressway and are quite different from the straight-line sections. The characteristics of traffic flow on road bends can be listed as follows.

(1) Significant Speed Difference. When traveling on road bends, due to the factors such as a driver's age, driving experience and his personality, and his familiarity with the road, together with the vehicle's performance, the vehicle speed on road bends varies considerably and the speed difference is significant between vehicles of different types and different models, and the mutual interaction between vehicles will be naturally serious.

(2) Longitudinal Gradient Exerts Greater Impact on Vehicles. In expressway design, the occurrence of longitudinal gradient exerts direct impact on large trucks: in climbing, the speed of the trucks is significantly lower, making its travel quite difficult; in downhill, the trucks are accelerated and are hard to decelerate, which is likely to be prone to traffic accidents and will have great impact on the vehicle operation and the road capacity.

(3) Degradation in the Vehicle Handling Performance. The friction between vehicle tires and road surface is used as the centripetal force needed for vehicle traveling on road bends. The centripetal force will lead to the lateral offset of vehicles, thereby increasing the requirements of handling performance in direction. The higher the vehicle speed is, the more difficult it is to manipulate the vehicle.

(4) Reduced Driving Comfort. When traveling on road bends, the lateral force coefficiency increases, resulting in the reduction of the vehicle stability; when the lateral force is too large, the occupants will be discomforted; if the speed is too large, a rollover accident will possibly occur. 


\section{CA Model of Single-Lane Bends}

3.1. Model Establishment. A CA model of a bend on a singlelane is established in this work, as shown in Figure 1: the lane of this model is composed of a total of $L$ (set $L=7000$ in this work) cells, and the length of each cell is $1 \mathrm{~m}$; namely, the actual length of the lane in the model is $7 \mathrm{~km}$. In the initial moment, vehicles are equally spaced on the lane. The length of each vehicle is $L_{\text {car }}$; vehicle speed on the bend is $V_{\text {safe }}$; the maximum velocity for the vehicles $V_{\max }=35$ cells $/ \mathrm{s}$ $=126 \mathrm{~km} / \mathrm{h}$; curve length is $L_{\text {bend }}$. The bend is set at the intermediate position of the lane, the vehicle deceleration is $b$, the friction coefficiency of the lane is $\mu$, the gravity acceleration is $g$, and the curve radius is $r$. Periodic boundary condition can be adopted in this model; that is, when a vehicle running out of the $L$ th cell, it will enter the system again from the first cell. It can be inferred from Figure 1 that the lane can be divided into straight-line section, transition section, and the bend section. The revolution laws are different for a vehicle when it is on different sections. On the straight-line section, because the diver is in a relaxed state, his driving behaviors such as sudden shift or change lanes are unlikely to occur. On the transition section, a vehicle may be decelerated until entering the bend due to the fact that a driver must adjust himself to the vehicle state according to his driving experience and the vehicle's running speed, and the sharp deceleration may occur at a higher vehicle speed, leading to the increased uncertainty on road safety. After entering the bend, the vehicle needs to turn around the bend, and the driver is normally driving cautiously, together with the case where vehicle speed is typically reduced before entering the bend. Therefore, the vehicle will be accelerated to the safe speed after entering the bend to pass through as quickly as possible. Different evolutionary rules are developed for specific driving behaviors on different sections, which can reflect the driving state more realistically.

Before the enactment of the model operating rules, the following assumptions are made in this paper accordingly.

(1) The external environment is good, without interference of pedestrians or horizontal traffic, and the effect of weather and other factors is ignored hereby.

(2) On the straight-line section, the vehicles are running at the desired speed as possible, and vehicle speed will not increase after the vehicle reached its maximum speed.

(3) Vehicle speed is decelerated on the transition section before entering the bend.

(4) The speed of vehicles on the circular curve is not higher than the safe speed; namely, there is no accident caused by driving with too higher speed.

(5) No significant difference in vehicle performance is considered in this work; namely, the effects of acceleration performance, being empty, and with load are all ignored hereby.

3.2. Model Operating Rules. Based on the classic NaSch model, considering the characteristics of driving behavior on

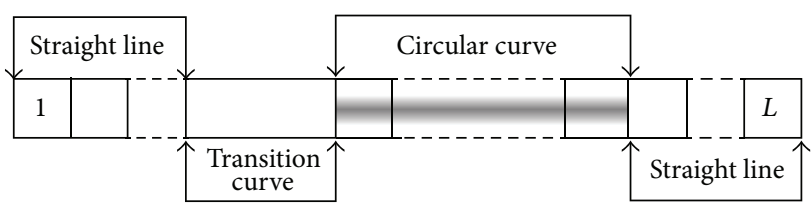

FIGURE 1: Structure diagram of the road bend.

the bend, the effects of road bend conditions (curve radius $r$, curve arc length $s$, and road surface friction coefficient $\mu$ ) and vehicle characteristics on the vehicle speed and latency probability, the evolution rules of the model in this paper are summarized accordingly, which include the evolution rules of normal sections, transition sections and bend sections, which can be detailed below.

\section{(1) Evolution Rules of Normal Sections}

Step 1 (acceleration). Consider

$$
V n(t)=\min \left(V n(t)+1, V_{\max }\right) .
$$

Step 2 (deceleration). Consider

$$
V n(t)=\min \left(V n(t), \operatorname{gap}_{n}(t)\right) .
$$

Step 3 (randomization deceleration with probability P10). Consider

$$
V n(t)=\max (V n(t)-1,0)
$$

Step 4 (location update). Consider

$$
X n(t)=X n(t)+V n(t) .
$$

\section{(2) Evolution Rules of Transition Sections}

Step 1 (acceleration). If $V n(t)<V_{\text {expect }}$, then vehicle will be accelerated with probability $P 2$ as

$$
V n(t)=V n(t)+D 1 .
$$

Step 2 (deceleration). If $V n(t)>V_{\text {expect }}$, then vehicle will be decelerated with probability $P 3$ as

$$
V n(t)=V n(t)-D 2 .
$$

Here $V_{\text {expect }}=\left(V n^{2}-V_{\text {safe }}^{2}\right) / 2 b$ is the desired running speed on the transition section, which is considered as reference to reduce the sharp shift of vehicle speed. When the vehicle speed is less than $V_{\text {expect }}$, the vehicle will be accelerated (the acceleration is $D 1$ ) with probability $P 2$; when the vehicle speed is larger than $V_{\text {expect }}$, the vehicle will be decelerated (the acceleration is D2) with probability $P 3$.

Step 3 (safe deceleration). Consider

$$
V n(t)=\min \left(V n(t), \operatorname{gap}_{n}(t)\right) .
$$

Step 4 (deceleration with probability P11). Consider

$$
V n(t)=\max (V n(t)-1,0) .
$$


Step 5 (location update). Consider

$$
X n(t)=X n(t)+V n(t) .
$$

(3) Evolution Rules of Bend Sections

Step 1 (acceleration). If $V n(t)<V_{\text {safe, }}$ then vehicle will be accelerated with probability $P 4$ as

$$
V n(t)=\min \left(V n(t)+1, V_{\text {safe }}\right) .
$$

Here this means that the vehicle will be accelerated with probability $P 4$ when the vehicle speed is less than the safe speed.

Step 2 (forced deceleration). If $V n(t)>V_{\text {safe }}$, then the vehicle must be decelerated

$$
V n(t)=V_{\text {safe }} .
$$

When the vehicle enters the bend, if the vehicle speed is greater than the safe speed, to ensure driving safety, the vehicle speed must be decelerated to the safe speed; otherwise, the vehicle may be skidded off the lane because of the excessive centrifugal force.

Step 3 (safe deceleration). Consider

$$
V n(t)=\min \left(V n(t), \operatorname{gap}_{n}(t)\right) .
$$

Step 4 (randomization deceleration with probability P12). Consider

$$
V n(t)=\max (V n(t)-1,0) .
$$

Step 5 (location update). Consider

$$
X n(t)=X n(t)+V n(t) .
$$

Here $V n(t)$ is the speed of vehicle $n$ at time $t$; $X n(t)$ is the

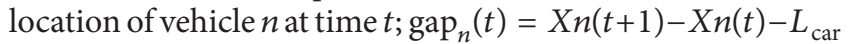
is the distance between vehicle $n$ and the front vehicle at time $t$; $V_{\text {safe }}$ is the safety speed (also the maximum speed) on the bend; $P 10, P 11$, and $P 12$ are the randomization deceleration probability for normal sections, transition sections, and bend sections, respectively; $P 2$ is the deceleration probability when the vehicle speed is larger than the desired speed on the transition section; $P 3$ is the acceleration probability when the vehicle speed is less than the desired speed on the transition section; $P 4$ is the acceleration probability when the vehicle speed is less than the maximum speed on the bend section.

\section{Simulation Results}

In this section, we want to conclude the rule of effects of road bend conditions and vehicle characteristics on the vehicle speed and latency probability. For the model building, we give the following parameter for the simulation.

Set $L_{\text {car }}=7$ cells, $g=10 \mathrm{~m} / \mathrm{s}^{2}, P 10=0.15, P 11=0.2, P 12=$ $0.1, P 2=0.3, P 3=0.1, P 4=0.2, D 1=2$ cells, $D 2=1$ cells, simulation steps $T_{\text {step }}=20000$, and Bendpoint $=L / 2$ (the bend is located on the middle of the lane). The average value of 20 simulation results is adopted to eliminate the impact of randomness of the results.

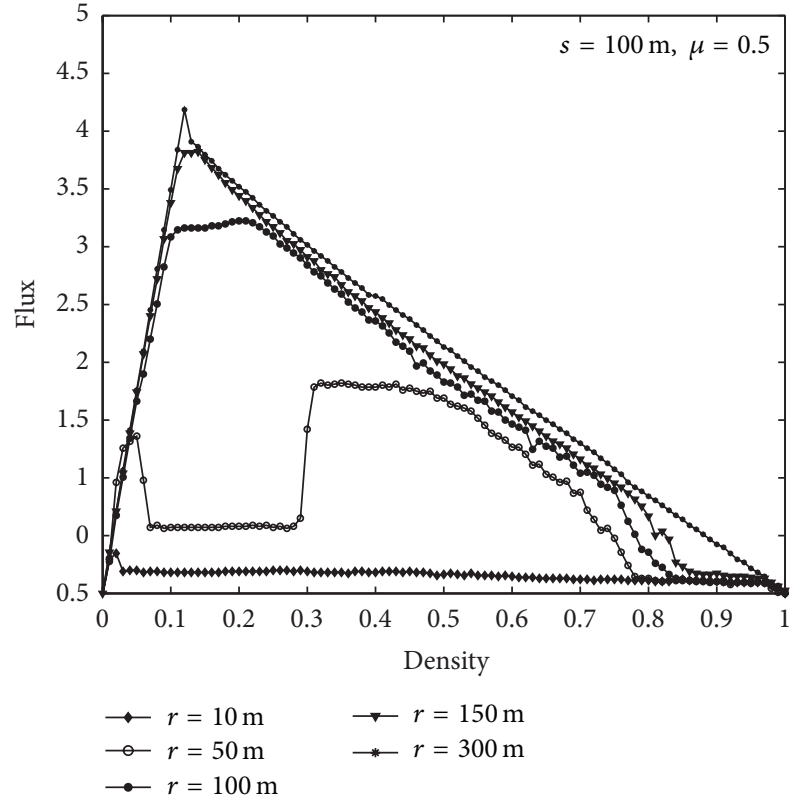

FIGURE 2: The fundamental diagram under different radii when $s=$ $100, \mu=0.5$.

4.1. The Effect of Bend Radius on Traffic Flow. Road bend is the critical factor among all the road alignment elements, which is determined by its radius. Therefore, the effect of bend radius on traffic flow is discussed in this paper firstly, and the fundamental diagram is shown in Figure 2.

In this paper, set the curve length $s=100 \mathrm{~m}$, the road surface friction coefficient $\mu=0.5$, and the curve radius $r$ $=10 \mathrm{~m}, 50 \mathrm{~m}, 100 \mathrm{~m}, 150 \mathrm{~m}$, and $300 \mathrm{~m}$. As can be seen from Figure 2, with the increase of the bend radius, the peak flow will be continuously increased, and the fundamental diagram will become more similar to that of the classic NaSch model; when the bend radius is greater than $150 \mathrm{~m}$, the densityflow diagram is close to the classic NaSch model; when the radius is greater than $300 \mathrm{~m}$, the density flow is consistent with the classic NaSch model; for relatively small bend radius, a "plateau" that does not change with density will appear, and the smaller the radius, the longer the "plateau" phase; when the bend radius is not so small (greater than $50 \mathrm{~m}$ ), the density-flow diagram is close to the classic NaSch model on both sections separated by the proposed "plateau."

Firstly, the appearance of the "plateau" is because of the inhibition effect of the road bend. The smaller the bend radius, the smaller the maximum vehicle's speed on the bend, resulting in a blockage around the bend. The phenomenon of go-and-stop before entering the bend hinders the transformation from crowded state to free-flow, leading to a long "plateau" phase. In this case, the bend can be regarded as a "bottleneck" in the road, which is similar to toll stations or other facilities on an expressway. For small density, the traffic flow will be increased with the increase of the density; for large density, the traffic flow will be decreased with the increase of the density. For small density, the interaction between vehicles is weak, so the vehicles can remain in free 


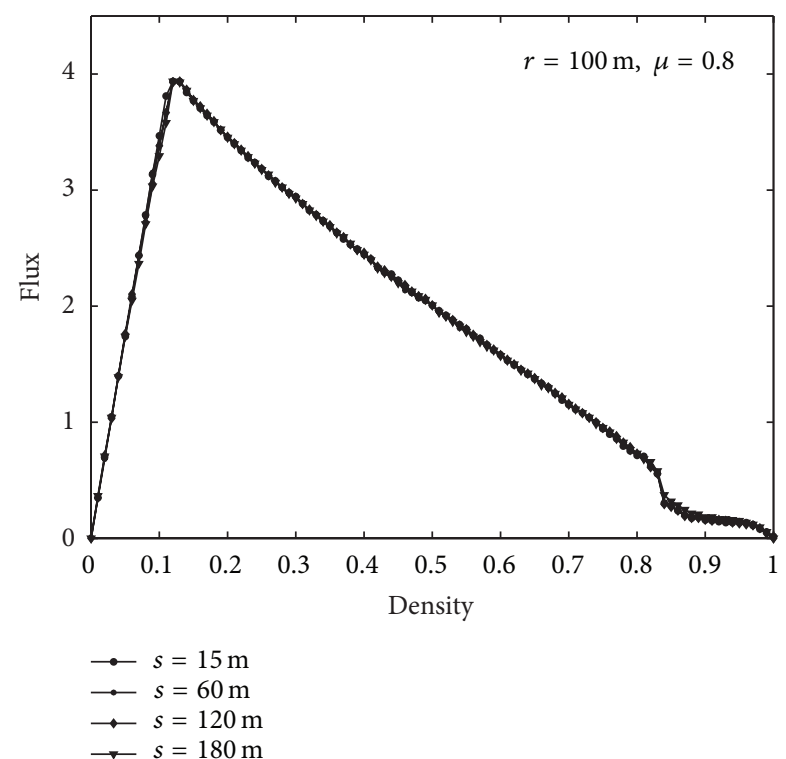

FIgURE 3: The fundamental diagram under different bend arc lengths when $r=100 \mathrm{~m}, \mu=0.8$.

state before and after passing through the bend, and the "bottleneck" effect was not so significant. For large density, the interaction between vehicles is quite strong, and the vehicles are in a congested state, so the inhibition effect of the bend is secondary compared to the interaction between the vehicles. Therefore, the main factor affecting the traffic flow is density, and the fundamental diagram exhibits a linear relationship.

Figures 3 and 4 show the density-flow fundamental diagrams under different bend arc lengths $(s=15 \mathrm{~m}, 60 \mathrm{~m}, 120 \mathrm{~m}$, and $180 \mathrm{~m}$ ). As can be seen from Figure 3, the difference between the fundamental diagram and the classic NaSch model is small under different bend arc lengths. For small density, the longer the length, the smaller the traffic flow; for large density, the fundamental diagrams are basically overlapped. In Figure 4, a "plateau" that does not change with density appears in the fundamental diagrams under different bend arc lengths, indicating the effect of bend arc length on traffic flow, will be smaller than that of the bend radius.

\subsection{The Effect of Road Surface Friction Coefficiency on Traffic} Flow. Road surface friction coefficiency plays an important role in highway engineering design, which not only determines the road passing capacity but also has an important influence on the vehicle driving safety. The value of friction coefficiency is determined by the following factors: tire size, tire pressure, wheel load, road construction, road conditions, and the vehicle's speed, as well as the service time of the road surface.

Figure 5 shows the density-flow fundamental diagrams under different friction coefficiency $(\mu=0.2,0.5,0.7,0.8$, and 0.9 ). As can be seen from the figure, the fundamental diagrams under different friction coefficiency exhibit the same trend with fundamental diagrams under different radii,

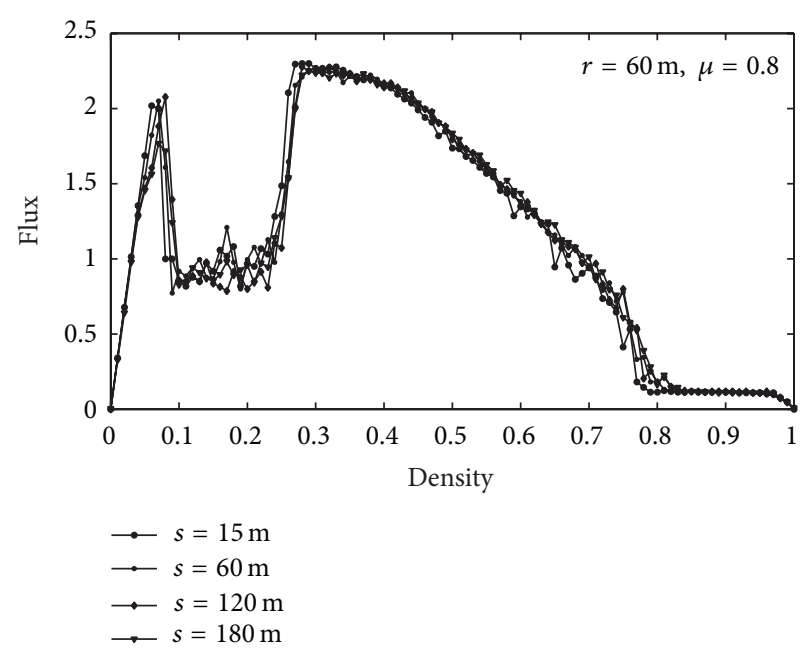

FIgURE 4: The fundamental diagram under different bend arc lengths when $r=60 \mathrm{~m}, \mu=0.8$.

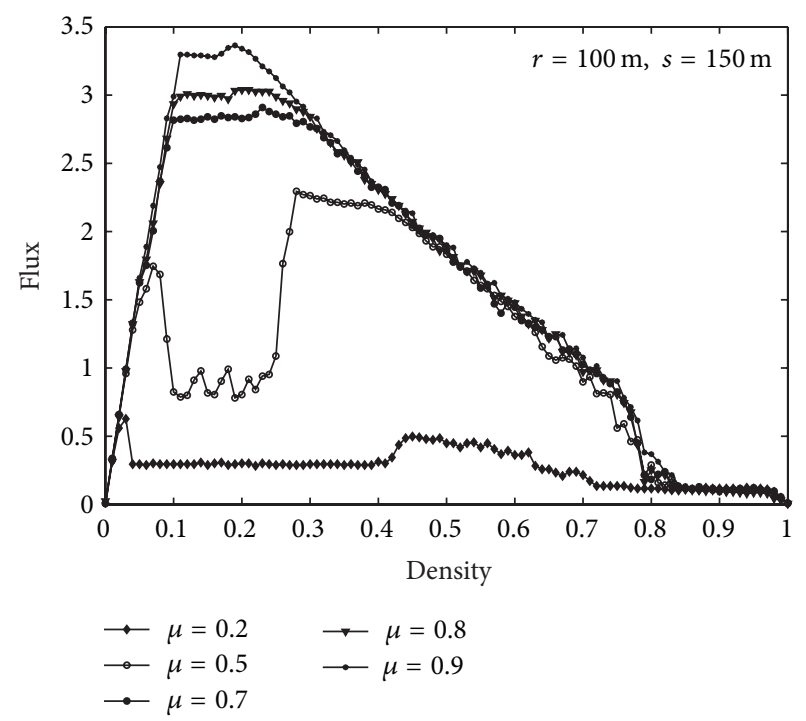

FIGURE 5: The fundamental diagram under different friction coefficients when $r=100 \mathrm{~m}, s=150 \mathrm{~m}$.

indicating that the effect of friction coefficiency is consistent with the impact of radius. Under the same bend radius, the larger the friction coefficient, the greater the limit speed $V_{\text {safe }}$ and the weaker the inhibition and "bottleneck" effect of the bend; therefore, the road passing capacity and the traffic flow are improved with the increase of the friction coefficiency.

\section{Conclusions}

Road bend is an important part of the road. In this paper, we want to find the structure and characteristics of road bends; based on this, a single-lane CA model with bends is established accordingly. Using this model, the traffic flow is explored under different bend radii; bend arc length, and road friction coefficiency. Simulation results show that a bend has 
an inhibitory effect on traffic flow, in which the impact of bend radius and road surface friction coefficiency is more significant than that of the bend arc length. The results can help readers to better understand the effects of road bends on traffic flow and help traffic engineers to reasonably design the bend radius.

\section{Conflict of Interests}

The authors declare that there is no conflict of interests regarding the publication of this paper.

\section{Acknowledgments}

This work is supported by the Humanities Social Sciences Programming Project of the Ministry of Education of China nos. 10YJA630126, 12YJC630200, 12YJC630100, the State Social Science Fund Project no. 11CJY067, and the Natural Science Foundation of Gansu Province, China, no. 1107RJYA070 and 1208RJZA164.

\section{References}

[1] S. Wolfram, "Statistical mechanics of cellular automata, Reviews of Modern Physics, vol. 55, no. 3, pp. 601-644, 1983.

[2] K. Nagel and M. Schreckenberg, "A cellular automaton model for freeway traffic," Journal de Physique I, vol. 2, pp. 2221-2229, 1992.

[3] Y. Ishibashi and M. Fukui, "Traffic flow in ID cellular automaton model including cars moving with high speed," Journal of the Physical Society of Japan, vol. 65, no. 6, pp. 1868-1870, 1996.

[4] O. Biham, A. A. Middleton, and D. Levine, "Self-organization and a dynamical transition in traffic-flow models," Physical Review A, vol. 46, no. 10, pp. R6124-R6127, 1992.

[5] Y. S. Qian, W. J. Li, J. W. Zeng, M. Wang, J. W. Du, and X. P. Guang, "Cellular automaton models of highway traffic flow considering lane-control and speed-control," Communications in Theoretical Physics, vol. 56, no. 4, pp. 785-790, 2011.

[6] S. C. Yang, C. Deng, T. Q. Tang, and Y. S. Qian, "Electric vehicle's energy consumption of car-following models," Nonlinear Dynamics, vol. 71, no. 1-2, pp. 323-329, 2013.

[7] T. Q. Tang, H. J. Huang, and S. G. Zhao, "A signal light model and its stability analysis," International Journal of Modern Physics B, vol. 24, no. 28, pp. 5613-5623, 2010.

[8] T. Q. Tang, P. Li, Y. H. Wu, and H. J. Huang, "A macro model for traffic flow with consideration of static bottleneck," Communications in Theoretical Physics, vol. 58, no. 2, pp. 300306, 2012.

[9] T. Q. Tang, Y. Li, and H. J. Huang, "The effects of taxi on traffic flow," International Journal of Modern Physics C, vol. 20, no. 10, pp. 1537-1546, 2009.

[10] T. Q. Tang, H. J. Huang, and H. Y. Shang, "A dynamic model for the heterogeneous traffic flow consisting of car, bicycle and pedestrian," International Journal of Modern Physics C, vol. 21, no. 2, pp. 159-176, 2010.

[11] H. X. Ge, H. B. Zhu, and S. Q. Dai, "Cellular automaton traffic flow model considering intelligent transportation system," Acta Physica Sinica, vol. 54, no. 10, pp. 4621-4626, 2005.
[12] H. X. Ge and R. J. Cheng, "The "backward looking" effect in the lattice hydrodynamic model," Physics A, vol. 387, no. 28, pp. 6952-6958, 2008.

[13] H. L. Zhou, Z. Y. Gao, and K. P. Li, "Cellular automaton model for moving-like block system and study of train's delay propagation," Acta Physica Sinica, vol. 55, no. 4, pp. 1706-1710, 2006.

[14] Y. P. Fu, Z. Y. Gao, and K. P. Li, “The characteristic analysis of the traffic flow of trains in speed-limited section for fixed-block system," Acta Physica Sinica, vol. 56, no. 9, pp. 5165-5171, 2007.

[15] K. P. Li, Z. Y. Gao, and B. Ning, "Modeling fixed-block railway signaling system using cellular automata model," International Journal of Modern Physics C, vol. 16, no. 11, pp. 1793-1801, 2005.

[16] K. P. Li, Z. Y. Gao, and L. X. Yang, "Modeling and simulation for train control system using cellular automata," Science in China E, vol. 50, no. 6, pp. 765-773, 2007.

[17] S. K. Rao, "Unit curve for design of highway transitions," Journal of Transportation Engineering, vol. 121, no. 2, pp. 169-175, 1995.

[18] J. B. Bentley and W. E. Gallagher, "Review of highway curve design," Journal of the Institution of Highway Engineers, vol. 18, no. 11, pp. 7-16, 1971.

[19] E. Dabbour, S. Easa, and K. Raahemifar, "Optimum vertical curves for highway profiles using nonlinear optimization," in Proceedings of the Annual Conference: Canadian Society for Civil Engineering, pp. 2833-2841, 2008.

[20] B. Persaud, R. A. Retting, and C. Lyon, "Guidelines for identification of hazardous highway curves," Transportation Research Record, no. 1717, pp. 14-18, 2000.

[21] H. Mu and S. W. Yang, "Alignment design of curved section of highway," Journal of Chang'an University (Natural Science Edition), vol. 27, pp. 26-29, 2007.

[22] Y. J. Liang and Y. Xue, "Study on traffic flow affected by the road turning," Acta Physica Sinica, vol. 59, no. 8, pp. 5325-5331, 2010.

[23] M. Wang, J. W. Zeng, Y. S. Qian, and W. J. Li, "Properties of train traffic flow in a moving block system," Chinese Physics B, vol. 21, no. 7, Article ID 070502, 2012. 


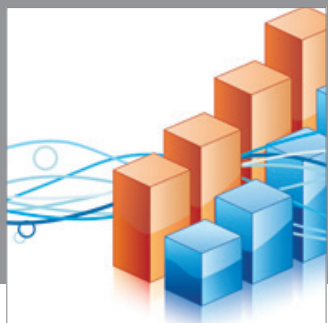

Advances in

Operations Research

mansans

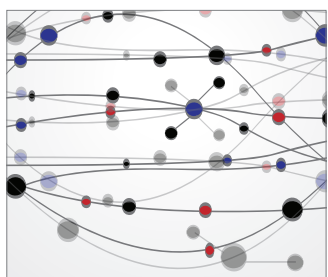

The Scientific World Journal
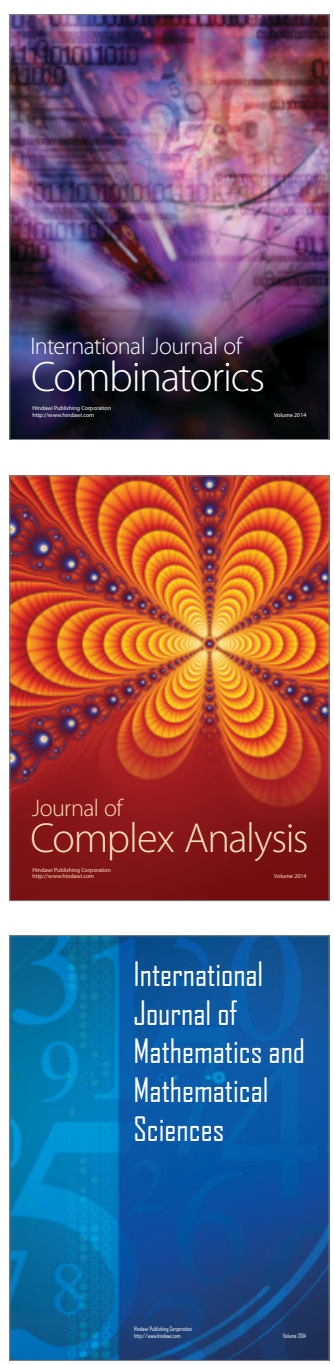
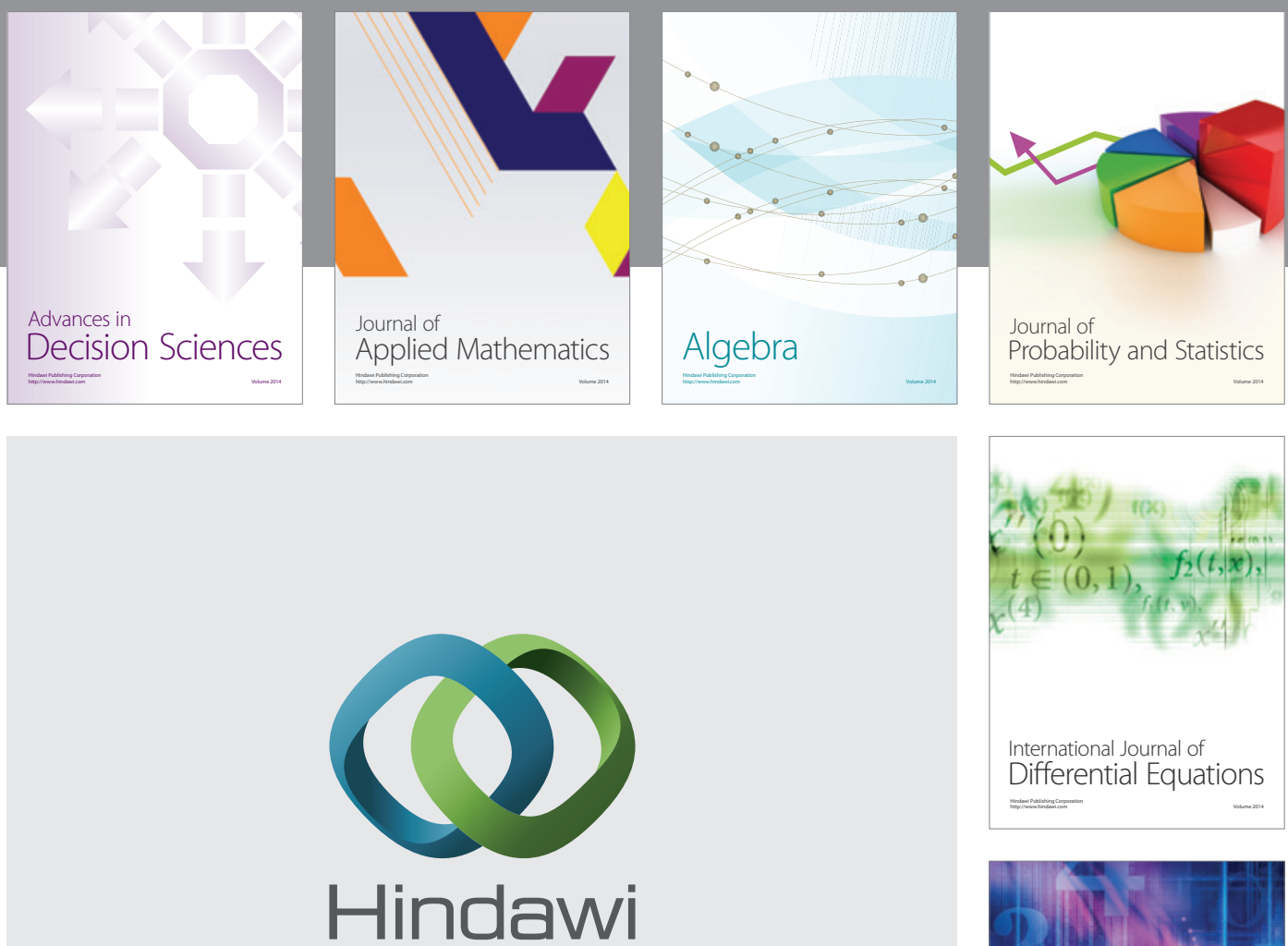

Submit your manuscripts at http://www.hindawi.com
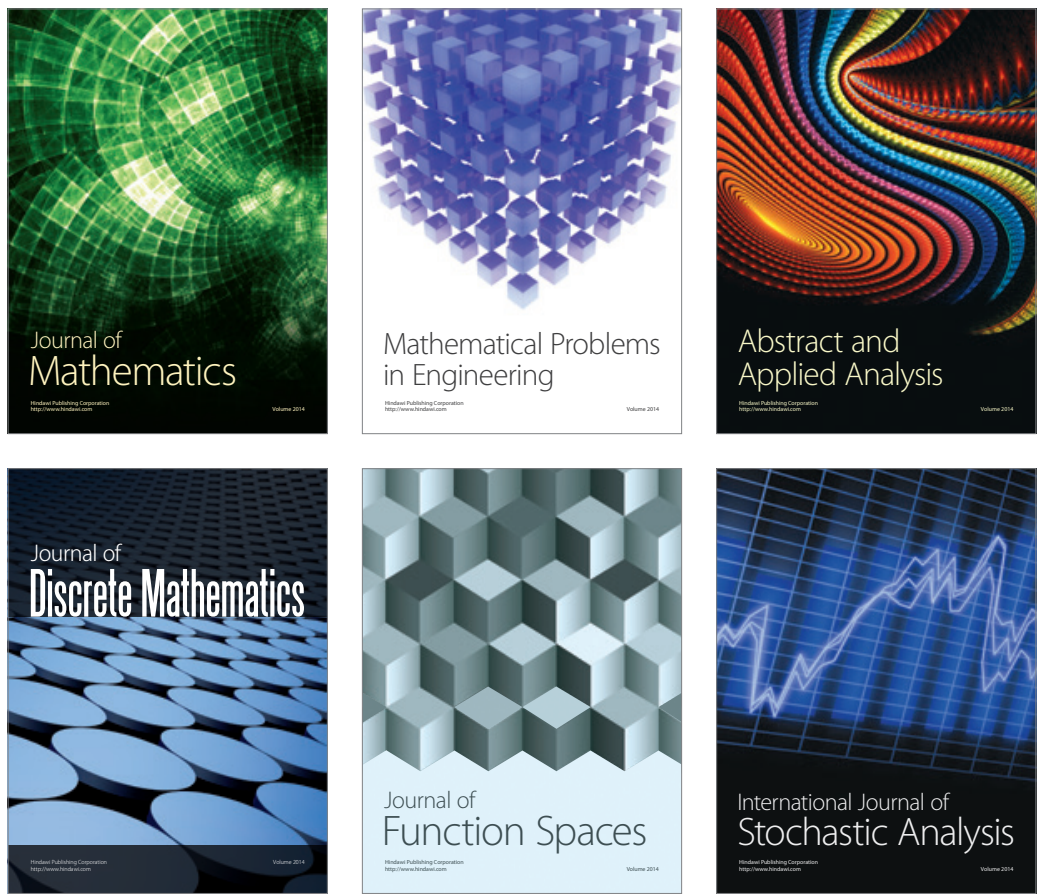

Journal of

Function Spaces

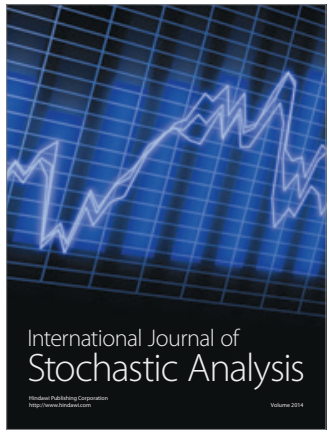

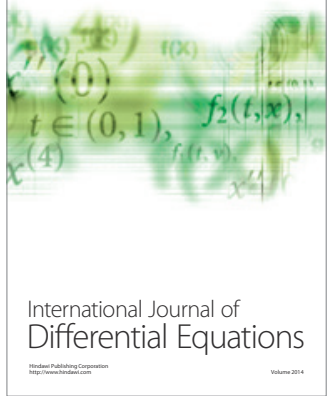
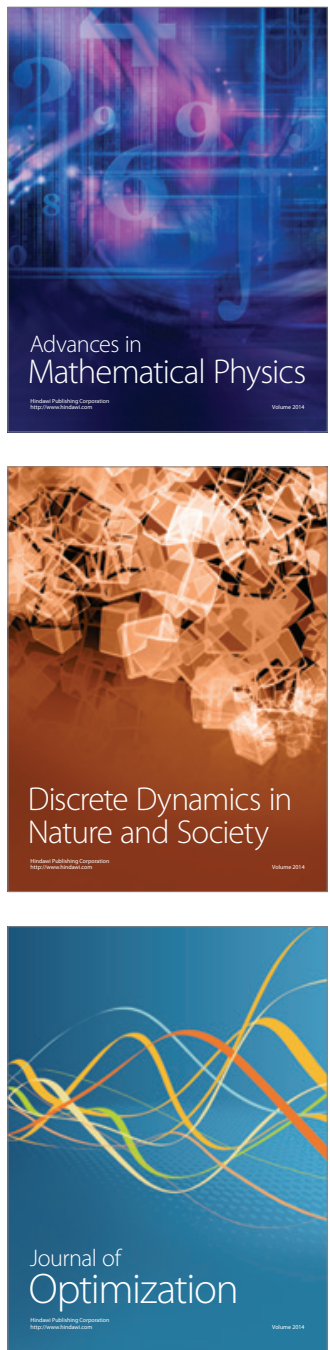